\title{
A cadaveric study of the variations in pulmonary fissures
}

\author{
Ekanayaka EADA ${ }^{1}$, Chrishan $\mathrm{AN}^{1}$, Dassanayake DMTD ${ }^{1}$, Dissanayake $\mathrm{JK}^{1}$ \\ ${ }^{I}$ Department of Anatomy, faculty of medicine, University of Peradeniya. \\ *eadaekanayaka@gmail.com
}

The right lung consists of three lobes separated by oblique and horizontal fissures and left lung consists of two lobes separated by a single oblique fissure. The knowledge on possible variations of pulmonary fissures is important in interpretation of radiological images and in pulmonary lobectomies or segmental resection. This study was carried out at the Department of Anatomy, Faculty of Medicine, University of Peradeniya, using 21 formalin fixed cadavers where routine dissections of the thorax was done by the medical undergraduates. Both lungs of each cadaver were removed and positioning of the pulmonary fissures and lobes were observed. Out of 21 pairs of right and left lungs, thirty three per cent of the right lungs and ninety per cent of the left lungs showed a typical presentation. Horizontal fissure was absent in twenty nine per cent of the right lungs and in thirty three per cent it was incomplete where it extended from the oblique fissure ending midway without extending up to the anterior border. The horizontal fissure was running low in one right lung enclosing a very small middle lobe. Small accessory fissures were present in two of the right and two of the left lungs. No sexual dimorphism noted in overall variations. The Present study revealed several variations where sixty six $\%$ of the right lungs had either an incomplete or absent horizontal fissure where the typical fissure pattern was seen less. Ten $\%$ of left lungs had accessory fissures. Such knowledge on variations is important during radiological interpretations and lung surgeries. 